\title{
Ultrasound-Accelerated Thrombolysis in High-Risk Perioperative Pulmonary Embolism: Two Case Reports and Review of Literature
}

Götz Schmidt ( $\sim$ goetz.f.schmidt@chiru.med.uni-giessen.de)

Justus Liebig Universitat Giessen https://orcid.org/0000-0003-2346-4265

Fabian Edinger

University of Giessen: Justus Liebig Universitat Giessen

Christian Koch

University of Giessen: Justus Liebig Universitat Giessen

Matthias Wolff

University of Giessen: Justus Liebig Universitat Giessen

Christoph Biehl

University of Giessen: Justus Liebig Universitat Giessen

Rüdiger Hörbelt

University of Giessen: Justus Liebig Universitat Giessen

Michael Sander

University of Giessen: Justus Liebig Universitat Giessen

\section{Case report}

Keywords: postoperative, surgical patients, surgery, embolectomy

Posted Date: September 30th, 2020

DOI: https://doi.org/10.21203/rs.3.rs-82629/v1

License: (a) This work is licensed under a Creative Commons Attribution 4.0 International License.

Read Full License 


\section{Abstract}

Background: Treatment of high-risk pulmonary embolism (PE) in perioperative patients remains challenging. Systemic thrombolysis is associated with a high risk of major bleedings and intracranial haemorrhage. High mortality rates are reported for open pulmonary embolectomy. Therefore, postoperative surgical patients may benefit substantially from catheter-directed ultrasound-accelerated thrombolysis (USAT).

Case summary: We report two cases of high-risk perioperative PE. Both patients developed severe haemodynamic instability leading to cardiac arrest. After the implantation of a veno-arterial extracorporeal membrane oxygenation (ECMO), they were both successfully treated with USAT. Adequate improvement of right ventricular function was achieved; thus, ECMO could be successfully weaned after three and four days, respectively. Both patients showed favourable outcomes and could be discharged to rehabilitation.

Discussion: The current European guideline on treatment of PE offers no specific therapies for perioperative patients with high-risk PE. However, systemic thrombolysis is often excluded due to the perioperative setting and the risk of major bleeding. Catheter-directed thrombolysis was shown to utilise less thrombolytic agent while obtaining comparable thrombolytic effects. The risk for major bleeding (including intracranial haemorrhage) is also significantly lowered.

Conclusion: Further prospective randomised controlled trials are necessary to determine the value of USAT treatment of high-risk PE in perioperative patients.

\section{Background}

Pulmonary embolism (PE) is a well-known clinical condition and may be related to increased morbidity and mortality. The Virchow's triad, consisting of stasis, endothelial injury and hypercoagulability, is fulfilled by different predisposing factors, which can be classified into patient- and setting-related risk factors [1-3]: Patient-related risk factors are usually permanent and include increasing age, arterial hypertension, congestive heart failure and cancer (with the highest risk in metastatic disease). Settingrelated risk factors are usually temporary and include not only fractures of the lower limb, hip or knee replacement, major trauma and blood transfusion, but also immobility and history of, and deep venous thrombosis (DVT) itself. The latter factors are of enormous importance in the perioperative environment, and - often, many of them often occur simultaneously.

For instance, the incidence of DVT in surgical patients has been reported to be between $2.7 \%$ and $9.6 \%$, while PE is thought to occur in 1.4\% - 3.2\% [4-6]. Identification of PE might be challenging: Dyspnoea, chest pain, syncope and haemoptysis represent unspecific symptoms. Thus, standardised scores like the revised Geneva clinical prediction rule were developed to assess the pretest-probability of PE [7]. PE generates increased right-ventricular afterload and reduced RV contractility. Thus, it may lead to an obstructive shock with reduced RV function and consecutive reduced left-ventricular output [3]. This may 
result in sudden haemodynamic instability and cardiac arrest, requiring immediate medical treatment. RV failure and haemodynamic instability both increase the early postoperative mortality [8]. The pulmonary embolism severity index (PESI) [9] has been developed to assess the mortality risk in patients with PE. Age, male sex, cancer, chronic heart failure, pulse rate $\geq 110$ beats per minute, systolic blood pressure < $100 \mathrm{mmHg}$, respiratory rate $>30$ breaths per minute, temperature $<36^{\circ} \mathrm{C}$, altered mental status and arterial haemoglobin saturation $<90 \%$ are considered. The predicted mortality, which is lowest in Class I $(0 \%-1.6 \%)$ and highest in Class V $(10 \%-24.5 \%)$, may lead to an appropriate, individual therapy. According to the European Guidelines for the diagnosis and management of acute PE, the primary treatment goal in high-risk PE patients is to maintain haemodynamic and respiratory stability [3]. Therefore, oxygen supply and non-invasive or invasive mechanical ventilation may be necessary. RV failure and subsequent systemic hypotension should be treated with volume optimisation, vasopressors and inotropes. Especially after high-risk PE with cardiac arrest, veno-arterial extracorporeal membrane oxygenation (VA-ECMO) may be used to achieve haemodynamic stability and to guarantee adequate tissue oxygenation [10]. To prevent further thrombus formation, all patients should receive therapeutic anticoagulation. Initially anticoagulation may be achieved with heparin or fondaparinux. Recently, the new oral anticoagulants rivaroxaban and apixaban have also been shown to be non-inferior in this cohort of patients [3].

However, particularly in high-risk PE, additional causal therapy is needed; therefore, different reperfusion strategies exist. Systemic thrombolysis (Class I recommendation) may be achieved by using fibrinolytic agents like recombinant tissue-type plasminogen activator (rtPA), streptokinase or urokinase [3]. These reduce RV dilatation through reduction of pulmonary obstruction, pulmonary artery pressure and pulmonary vascular resistance. Although reduced mortality rates could be shown in high-risk PE [11], unsuccessful systemic thrombolysis may occur in up to $8 \%$ [12]. In addition, concerns may arise regarding the risk of severe bleeding and intracranial haemorrhage [13]. Surgical pulmonary embolectomy (Class I recommendation for patients, in whom systemic thrombolysis is contraindicated or has failed) is performed via an open access to the two main pulmonary arteries and the consecutive removal of obstructive clots [3]. Therefore, cardioplegic cardiac arrest with cardiopulmonary bypass is usually necessary. In comparison to systemic thrombolysis, Lee et al. reported similar survival rates and a lower risk of stroke [14]. However, surgical embolectomy showed the highest mortality rates (32\%) after cardiac arrest [15].

In addition to systemic thrombolysis and surgical revascularisation, percutaneous catheter-directed therapy may also be considered as a Class Ila recommendation [3]: Via the femoral or jugular vein, a catheter is brought to the pulmonary artery where aspiration thrombectomy or local low-dose thrombolysis is performed [16]. Furthermore, ultrasound-assisted thrombolysis devices deliver ultrasound to ensure an increase in the clots' surface area and therefore improve the effect of the low-dose thrombolytic agent [17]. The management of perioperative patients with high-risk PE is challenging. According to current guidelines, major surgery is a relative exclusion criterion for systemic thrombolysis [3]. Surgical embolectomy must also be considered with caution, as some studies report rather high mortality rates and another major surgery including extracorporeal circulation has to be performed. The 
percutaneous catheter-directed approach may be an attractive option in haemodynamically unstable patients suffering from PE after major surgery. We report the first treatment - to our knowledge - of perioperative high-risk PE leading to cardiac arrest and VA-ECMO implantation with ultrasoundaccelerated catheter-directed thrombolysis (USAT).

\section{Case Summary}

\section{Case 1}

A 59-year-old female with a body mass index (BMI) of $28.4 \mathrm{~kg} / \mathrm{m}^{2}$ underwent elective cemented knee replacement surgery. With a medical history of arterial hypertension, intraoperative analgesia was achieved with spinal anaesthesia (3.4 ml of bupivacaine $0.5 \%)$ and sedation with propofol $(2.4 \mathrm{mg} / \mathrm{kg} / \mathrm{h}$ ). During the first three postoperative days (POD), her recovery proceeded without any abnormalities. Prophylactic anticoagulation was achieved with weight-adjusted low-molecular weight heparin (LMWH, certoparin 3000 I.E. s.c. per day). On POD 4 the patient developed dyspnoea during mobilisation. The subsequent CT scan revealed bilateral PE; therefore, the patient was transferred to the intermediate care unit. On admission, the patient was awake, responsive and did not present dyspnoea in supine position. Anticoagulation was performed with a bolus of 5000 I.E. of heparin, followed by enoxaparin $(2 \times 0.8 \mathrm{mg}$ s.c. per day). In good overall condition and with a corresponding PESI-Score of 59 (Class I, mortality risk $0 \%-1.6 \%$ ), the patient was readmitted to the general ward in the morning of POD 5. In the evening, the patient collapsed again and presented with dyspnoea and tachycardia. Transthoracic echocardiography revealed severe RV dilatation (Fig. 1a), tricuspid regurgitation, decreased tricuspid annular plane systolic excursion (TAPSE, $11 \mathrm{~mm}$ ) and flattened interventricular septum (Fig. 1b). Due to acute cardiac decompensation and pulseless ventricular tachycardia, cardiopulmonary resuscitation (CPR) and intubation had to be performed. Haemodynamic stabilisation could only be achieved after implantation of a VA-ECMO. Noradrenaline was given with a maximum rate of $0.06 \mu \mathrm{g} / \mathrm{kg} / \mathrm{min}$. Levosimendan $(0.1 \mu \mathrm{g} / \mathrm{kg} / \mathrm{min})$ ensured additional inotropic support. The subsequent angiography showed bilateral occlusion of the main pulmonary arteries (Fig. 2).

According to the PESI-Score, the patient was now classified in Class V (very high mortality risk, $10 \%$ $24.5 \%)$, underlining the need for urgent reperfusion therapy. However, due to the high bleeding risk after major surgery and according to the European guideline, systemic thrombolysis was relatively contraindicated. The possibility of surgical embolectomy was withdrawn because of the reported high mortality rates after cardiac arrest and due to the highly invasive extent of the procedure. As a consequence of the interdisciplinary team discussion, involving cardiothoracic surgeons, cardiologist, radiologists and intensive care physicians, ultrasound-accelerated catheter-directed thrombolysis (EkoSonic Endovascular System [EKOS Corporation, a Boston Scientific Company, Bothell, WA, USA]) was performed. Via the right femoral vein one catheter was inserted into the right pulmonary artery. Subsequently, another catheter was brought to the left pulmonary artery via the left jugular vein (Fig. 3). After the ultrasound emission, each catheter released $1 \mathrm{mg} / \mathrm{h}$ of rtPA during the first five hours, followed 
by $0.5 \mathrm{mg} / \mathrm{h}$ rtPA for ten hours. Therapeutic anticoagulation was now established with argatroban $(0.5 \mu \mathrm{g} / \mathrm{kg} / \mathrm{min})$ and partial thromboplastin time (aPTT) was targeted at $60-70 \mathrm{~s}$. Due to stabilisation of the RV-function, ECMO support was ended three days after the implantation. During that time, the patient received $1.5 \mathrm{I}$ red blood cell concentrate, $0.4 \mathrm{I}$ fresh frozen plasma and $0.4 \mathrm{I}$ albumin $20 \%$. A further CT scan revealed regression of thrombotic burden (Fig. 4), showing only small clots in the lobar arteries. Due to respiratory and haemodynamic stabilisation, the patient was extubated one day later. Subsequently, the therapeutic anticoagulation was changed to an oral treatment with apixaban $(2 \times 10 \mathrm{mg}$ p.o. for one week, $2 \times 5 \mathrm{mg}$ p.o. subsequently). Ten days later, the patient was transferred to the general ward, from which she was discharged to ambulatory treatment in good condition after nine days.

\section{Case 2}

A 59-year-old female with a BMl of $23.5 \mathrm{~kg} / \mathrm{m}^{2}$ suffering from renal cell carcinoma (RCC) metastasis in the right lobe of the liver, underwent elective right hemihepatectomy. Medical history included RCC that was treated with nephrectomy, splenectomy and pancreatic left resection in 2003. Currently, the patient presented in a good condition. After the uneventful surgery she was transferred to the intensive care unit. Intermittent pneumatic compression (Kendall SCD 700, Cardinal Health, Ireland) was used as deepvenous thrombosis prophylaxis. Prophylactic anticoagulation was first achieved with heparin (400 I.E./h) and was subsequently changed to certoparin (3000 I.E. s.c. per day). Her recovery proceeded without any abnormalities; thus, she was discharged to the general ward at POD 1. One day later, she collapsed during mobilisation and was immediately transferred to the radiology department. On the way, she became haemodynamically unstable and CPR was started. Orotracheal intubation was performed and the patient was transferred to the intensive care unit, where a VA-ECMO was implanted. After haemodynamic stabilisation, a CT scan was performed and revealed bilateral massive PE spreading through all lobar and segmental arteries. Due to the large thrombotic burden and a massive derangement of coagulation, an interdisciplinary decision, involving cardiothoracic and general surgeons, cardiologists, radiologists and intensive care physicians, was made for interventional treatment. Pulmonary angiography and consecutive catheter implantation (EkoSonic Endovascular System [EKOS Corporation, a Boston Scientific Company, Bothell, WA, USA]) in each pulmonary artery allowed USAT. The left pulmonary artery catheter was inserted through the left femoral vein, and the right pulmonary artery was reached through the right jugular vein. During the following 12 hours, $12 \mathrm{mg}$ of rtPA were administered $(0.5 \mathrm{mg} / \mathrm{h} /$ catheter). After 6 hours, an additional CT scan already revealed partial recanalisation of the lobar arteries. Noradrenaline was administrated at a maximum of $0.19 \mu \mathrm{g} / \mathrm{kg} / \mathrm{min}$ and inotropic support was delivered using low-dose dobutamine ( $3 \mu \mathrm{g} / \mathrm{kg} / \mathrm{min})$. During USAT with rtPA and therapeutic anticoagulation with argatroban (at a maximum of $0.46 \mu \mathrm{g} / \mathrm{kg} / \mathrm{min}$, targeted aPTT 60-80 s), a major abdominal bleeding from the operation's area occurred. The bleeding had to be treated in the operating theatre twice, and the patient received a total of 4.9 I red blood cell concentrate, 4.3 I fresh frozen plasma, 0.9 I platelet concentrate, $6 \mathrm{~g}$ fibrinogen, 1250 I.E. factor XII concentrate and 1500 I.E. prothrombin complex concentrate. Subsequently, examinations of RV function were performed with transoesophageal echocardiography (TEE). A daily improvement in biventricular function was recognised; as a result, the 
ECMO was removed after four days. Subsequently, the patient went through prolonged intensive care treatment, including tracheotomy, dialysis and complicated weaning. An empyema of the lower lobe due to a lung infarction-associated pneumonia had to be treated surgically before the patient's condition improved. She was transferred to the general ward 41 days after admission to the intensive care unit and was discharged to rehabilitation two weeks later.

\section{Discussion}

We report the first to two cases to our knowledge of perioperative high-risk PE including cardiac arrest that were treated by ultrasound-accelerated catheter-directed thrombolysis (USAT). Despite CPR, both patients' initial haemodynamic stabilisation was achieved by VA-ECMO therapy. Subsequently, successful thrombolysis with USAT led to favourable outcomes. Treatment of high-risk PE remains challenging. According to the European guideline for the management of acute PE, patients with high-risk PE should receive therapeutic anticoagulation and systemic thrombolysis (Class I recommendation). If systemic thrombolysis is contraindicated or has failed, surgical embolectomy should be performed (Class I recommendation). Percutaneous catheter-directed thrombolysis may be considered as second-line therapy (Class Ila recommendation). In combination with catheter-directed therapy or pulmonary embolectomy, the use of ECMO may be considered (Class IIb recommendation). Unfortunately, the guideline does not offer any specific strategies concerning the perioperative setting. It must be highlighted, that treatment options are strongly limited by the high bleeding risk during the perioperative period. Moreover, there are only a few reports regarding the treatment of perioperative high-risk PE. Some patients were solely treated with therapeutic anticoagulation and did not receive any further thrombolytic therapy $[18,19]$. Smith et al. reported a case of a perioperative submassive high-risk PE in the right upper lobe after hip hemiarthroplasty leading to cardiac arrest [18]. Return of spontaneous circulation was achieved after two minutes. Further therapy with anticoagulation and inotropic support with dobutamine led to adequate stabilisation; thus, thrombolytic therapy was withdrawn due to the high risk of bleeding. Hartmannsgruber et al. reported the successful application of systemic thrombolysis after high-risk perioperative PE in a patient with morbid obesity [20]. However, the patient had undergone umbilical hernia repair with a very low bleeding risk. Another patient undergoing major trauma surgery was treated with USAT after intraoperative massive high-risk PE [21]. Haemodynamic stabilisation was achieved with phenylephrine and fluid therapy. In contrast, both of our cases presented with cardiac arrest. Haemodynamic stabilisation could only be achieved by VA-ECMO underlining the immediate need for a reperfusion therapy that decrease mortality [11]. Cardiac arrest occurred in the presence of trained personnel; thus, high-efficiency CPR was started immediately. Compared to other reasons for cardiac arrest, patients surviving a high-risk $P E$ are reported to recover with favourable neurological outcomes [10]. VA-ECMO was shown to relieve the enormous RV afterload by bypassing the pulmonary vascular bed [22]. Nevertheless, there is a known association between ECMO and serious bleeding complications [23]. Additionally, in case 2, major bleeding requiring further surgery and transfusion of blood and coagulation products occurred during ECMO therapy. Coagulation products may be suspected to facilitate further thrombotic events, including oxygenator thrombosis. In adults, an $8.0 \%-12.9 \%$ rate of 
oxygenator clots during ECMO has been reported [24, 25]. Although it was shown that recombinant factor VIla can be safely used in ECMO patients, there are only limited data regarding other coagulation products [26]. Due to the additional risk of bleeding combined with a potential increased risk of thrombotic events, ECMO should be restricted to well-selected patients and experienced centres. In both patients, only low dose catecholamines were necessary: in case 1, Levosimendan was given not only to improve RV contractibility; rather it has also been shown to restore RV coupling by an additional pulmonary vasodilatation [27]. The perioperative period itself is associated with severe bleeding complications. After knee arthroplasty, significant blood loss frequently occurs during the postoperative period, with the highest values at POD 3 [28]. Major liver surgery is associated with severe complications not only due to the surgical site, but also because the impaired function of the remaining liver presents an obstacle. Jacquenod et al. reported a $53.5 \%$ rate of coagulopathy after major liver surgery. It must be highlighted that $84 \%$ of the patient's liver was resected in our reported case. This led to a significant impairment of coagulation and, subsequently, reduced secretory liver function. After resuscitation, the initial international normalised ratio was measured at 6.1, underlining the high risk for major bleeding and intracranial haemorrhage. Additionally, according to the ESC Guidelines on Acute Pulmonary Embolism, major surgery is a relative exclusion criterion for systemic thrombolysis [3]. The risk of bleeding complications - up to a $9.9 \%$ rate of severe bleedings and a $1.7 \%-5.0 \%$ rate of intracranial haemorrhage - is not negligible and must be considered carefully in the perioperative setting [11, 29]. Surgical thrombectomy is a highly invasive procedure and high mortality rates are reported [15]. However, it may be considered after unsuccessful systemic thrombolysis: Meneveau et al. reported improved outcomes after surgical embolectomy compared to repeat thrombolysis. Additionally, all bleeding events were fatal after repeated thrombolysis [12]. Therefore, our team decided that USAT was a feasible treatment strategy due to the high bleeding risk in our two presented cases. Further, USAT has been reported to be safe in non-surgical patients. Kuo et al. reported a significant improvement in mean pulmonary artery pressure and echocardiography after acute PE treated with catheter-directed therapy, with clinical success achieved in $85.7 \%$ of patients [30]. Tapson et al. showed an improved right ventricular-to-left ventricular diameter ratio and reduced embolic burden even when low-dose rtPA (8-24 mg) was administered [31]. Nevertheless, a $4 \%$ rate of major bleedings and one intracranial haemorrhage that was attributed to rtPA occurred. These encouraging results could be confirmed by Piazza et al., who reported only one severe bleeding among 150 patients [32]. A 10\% rate of moderate bleeding was observed after the cumulative admission of $24 \mathrm{mg}$ rtPA. Furthermore, no intracranial haemorrhage occurred. Kucher et al. performed a randomised controlled trial comparing USAT therapy with heparin only [33]. No major bleeding occurred in the intervention group after the admission of 10-20 mg rtPA. A higher overall risk of bleeding could not be detected. The additional use of ultrasound is thought to decrease treatment time and dose of the thrombolytic agent, which should therefore result in decreased morbidity and mortality [34]. Percutaneous catheter-directed treatment is associated with the lowest rate of major bleeding complications of all reperfusion strategies. We successfully used low-dose thrombolysis with a cumulative dose of $20 \mathrm{mg}$ in case 1 and $12 \mathrm{mg}$ in case 2 . Another meta-analysis confirmed that USAT results in a lower risk of major bleedings [35]. However, in case 2, major bleeding of the surgical site occurred during USAT therapy. Despite the high bleeding risk after major liver surgery, the thrombolytic agent administered may have 
accelerated the severity of bleeding, which led to massive transfusion and the need for further surgical therapy. In our cases, during rtPA-administration, TEE was performed periodically to investigate improvements in RV dilatation and RV function. This led to a clinically driven decision of whether a prolonged local thrombolysis was necessary. Thus, in comparison to systemic thrombolysis, the dose of thrombolytic agent applied could possibly be further reduced. Compared to systemic thrombolysis, currently available USAT devices require only $10 \%$ of the thrombolytic agent [36]. Due to possible bleeding complications and heparin-induced thrombocythemia (HIT), according to the 2019 ESC Guidelines for the diagnosis and management of acute $\mathrm{PE}$, unfractionated heparin (UFH) should be restricted to patients with high bleeding risk, haemodynamic instability or serious renal impairment [3]. Our first patient suffered from recurrent PE although therapeutic anticoagulation had been started only hours before. Additionally, the risk according to the PESI-score was very low. However, HIT tests revealed no pathological findings, although the test on tissue factor antibodies showed a marginally but insignificant result. As reported in our cases, further anticoagulation was achieved with argatroban. In comparison to UFH, argatroban was shown to significantly accelerate thrombolysis in vitro, despite no difference in PTT prolongation being seen [37]. With UFH, the same effect could only be seen with higher doses resulting in an over-prolonged PTT above $180 \mathrm{~s}$. Bethea et al. reported a case of recurrent PE after repeated thrombolysis in a patient with HIT [38]. While both patients were treated with USAT and argatroban, a fast improvement of the pulmonary artery pressure was monitored. In summary, we report two cases of highrisk perioperative PE in which USAT was performed. The immediate start of high-quality resuscitation led to a closely patient-adapted therapy with minimised application of thrombolytic agents and may therefore have helped to achieve favourable neurological outcomes. However, the European guideline for the management of acute PE does not offer any specific strategy for the treatment of perioperative patients. Further prospective randomised controlled trials are necessary to evaluate the value of adopted therapies including catheter-directed thrombolysis in perioperative patients suffering from high-risk PE.

\section{List Of Abbreviations}

aPTT - partial thromboplastin time

BMI - body mass index

CPR - cardiopulmonary resuscitation

DVT - deep venous thrombosis

ECMO - extracorporeal membrane oxygenation

HIT - heparin-induced thrombocythemia

LMWH - low-molecular weight heparin

PE - pulmonary embolism 
POD - postoperative day

RCC - renal cell carcinoma

rtPA - recombinant tissue-type plasminogen activator

TAPSE - tricuspid annular plane systolic excursion

TEE - transoesophageal echocardiography

UFH - unfractionated heparin

USAT - ultrasound-accelerated thrombolysis

VA-ECMO veno-arterial extracorporeal membrane oxygenation

\section{Declarations}

Ethics approval and consent to participate:

Not applicable.

Consent for publication:

Written consent for publication was obtained from both patients.

Availability of data and materials:

The datasets used during the current study are available from the corresponding author on reasonable request.

Competing interests:

The authors declare that they have no competing interests.

Funding:

FE received funding from the clinician scientist program of the Justus-Liebig-University Giessen.

Authors' contributions:

$\mathrm{GS}, \mathrm{FE}, \mathrm{CK}$ were involved in planning, data acquisition and writing the manuscript. $\mathrm{MW}, \mathrm{MS}, \mathrm{CB}$ and $\mathrm{RH}$ made substantial contributions to the conception and provided additional references and clinical experience to the discussion. All authors substantially revised it and have approved the submitted version. 
Not applicable.

\section{References}

1. Rogers MAM, Levine DA, Blumberg N, Flanders SA, Chopra V, Langa KM. Triggers of hospitalization for venous thromboembolism. Circulation. 2012;125:2092-9.

doi:10.1161/CIRCULATIONAHA.111.084467

2. Anderson FA, Spencer FA. Risk factors for venous thromboembolism. Circulation. 2003;107. doi:10.1161/01.CIR.0000078469.07362.E6

3. Konstantinides S V., Meyer G, Bueno H, Galié N, Gibbs JSR, Ageno W, et al. 2019 ESC Guidelines for the diagnosis and management of acute pulmonary embolism developed in collaboration with the European respiratory society (ERS). Eur Heart J. 2020;41:543-603. doi:10.1093/eurheartj/ehz405

4. Cook D, Crowther M, Meade M, Rabbat C, Griffith L, Schiff D, et al. Deep venous thrombosis in medical-surgical critically ill patients: Prevalence, incidence, and risk factors. Crit Care Med. 2005;33:1565-71. doi:10.1097/01.CCM.0000171207.95319.B2

5. Pan Y, Mei J, Wang L, Shao M, Zhang J, Wu H, et al. Investigation of the Incidence of Perioperative Pulmonary Embolism in Patients with Below-Knee Deep Vein Thrombosis after Lower Extremity Fracture and Evaluation of Retrievable Inferior Vena Cava Filter Deployment in These Patients. Ann Vasc Surg. 2019;60:45-51. doi:10.1016/j.avsg.2019.02.027

6. Park SJ, Kim CK, Park YS, Moon YW, Lim SJ, Kim SM. Incidence and factors predicting venous thromboembolism after surgical treatment of fractures below the hip. J Orthop Trauma. 2015;29:e349-54. doi:10.1097/BOT.0000000000000336

7. Le Gal G, Righini M, Roy PM, Sanchez O, Aujesky D, Bounameaux H, et al. Prediction of pulmonary embolism in the emergency department: The revised geneva score. Ann Intern Med. 2006;144:16571. doi:10.7326/0003-4819-144-3-200602070-00004

8. Harjola VP, Mebazaa A, Čelutkiene J, Bettex D, Bueno H, Chioncel O, et al. Contemporary management of acute right ventricular failure: A statement from the Heart Failure Association and the Working Group on Pulmonary Circulation and Right Ventricular Function of the European Society of Cardiology. Eur J Heart Fail. 2016;18:226-41. doi:10.1002/ejhf.478

9. Aujesky D, Obrosky DS, Stone RA, Auble TE, Perrier A, Cornuz J, et al. Derivation and validation of a prognostic model for pulmonary embolism. Am J Respir Crit Care Med. 2005;172:1041-6. doi:10.1164/rccm.200506-8620C

10. Yusuff HO, Zochios V, Vuylsteke A. Extracorporeal membrane oxygenation in acute massive pulmonary embolism: A systematic review. Perfus (United Kingdom). 2015;30:611-6. doi:10.1177/0267659115583377

11. Marti C, John G, Konstantinides S, Combescur C, Sanchez O, Lankeit M, et al. Systemic thrombolytic therapy for acute pulmonary embolism: A systematic review and meta-analysis. Eur Heart $\mathrm{J}$. 2015;36:605-14. doi:10.1093/eurheartj/ehu218 
12. Meneveau N, Séronde MF, Blonde MC, Legalery P, Didier-Petit K, Briand F, et al. Management of unsuccessful thrombolysis in acute massive pulmonary embolism. Chest. 2006;129:1043-50. doi:10.1378/chest.129.4.1043

13. Knuuti J, Wijns W, Capodanno D, Funck-brentano C, Denmark EP, Storey RF, et al. 2019 ESC Guidelines for the diagnosis and management of chronic coronary syndromes The Task Force for the diagnosis and management of chronic. 2019;1-71. doi:10.1093/eurheartj/ehz425

14. Lee T, Itagaki S, Chiang YP, Egorova NN, Adams DH, Chikwe J. Survival and recurrence after acute pulmonary embolism treated with pulmonary embolectomy or thrombolysis in New York State, 1999 to 2013. J Thorac Cardiovasc Surg. 2018;155:1084-1090.e12. doi:10.1016/j.jtcvs.2017.07.074

15. Wu MY, Liu YC, Tseng YH, Chang YS, Lin PJ, Wu TI. Pulmonary embolectomy in high-risk acute pulmonary embolism: The effectiveness of a comprehensive therapeutic algorithm including extracorporeal life support. Resuscitation. 2013;84:1365-70.

doi:10.1016/j.resuscitation.2013.03.032

16. Piazza G, Hohlfelder B, Jaff MR, Ouriel K, Engelhardt TC, Sterling KM, et al. A Prospective, Single-Arm, Multicenter Trial of Ultrasound-Facilitated, Catheter-Directed, Low-Dose Fibrinolysis for Acute Massive and Submassive Pulmonary Embolism: The SEATTLE II Study. JACC Cardiovasc Interv. 2015;8:1382-92. doi:10.1016/j.jcin.2015.04.020

17. Braaten J V., Goss RA, Francis CW. Ultrasound reversibly disaggregates fibrin fibers. Thromb Haemost. 1997;78:1063-8. doi:10.1055/s-0038-1657688

18. Smith D, Murauski J. Pulmonary Embolism in the Postanesthesia Care Unit: A Case Study. J Perianesthesia Nurs. 2017;32:6-14. doi:10.1016/j.jopan.2015.12.016

19. Mao Y, Wen S, Chen G, Zhang W, Ai Y, Yuan J. Management of intra-operative acute pulmonary embolism during general anesthesia: A case report. BMC Anesthesiol. 2017;17. doi:10.1186/s12871017-0360-0

20. Hartmannsgruber MWB, Trent FL, Stolzfus DP. Thrombolytic therapy for treatment of pulmonary embolism in the postoperative period: Case report and review of the literature. J Clin Anesth. 1996;8:669-74. doi:10.1016/S0952-8180(96)00145-6

21. Dudaryk R, Benitez Lopez J, Louro J. Diagnosis and Thrombolytic Management of Massive Intraoperative Pulmonary Embolism Guided by Point of Care Transthoracic Echocardiography. Case Rep Anesthesiol. 2018;2018:1-5. doi:10.1155/2018/8709026

22. Belohlavek J, Rohn V, Jansa P, Tosovsky J, Kunstyr J, Semrad M, et al. Veno-Arterial ECMO in severe acute right ventricular failure with pulmonary obstructive hemodynamic pattern. J Invasive Cardiol. 2010;22:365-9.

23. Thomas J, Kostousov V, Teruya J. Bleeding and Thrombotic Complications in the Use of Extracorporeal Membrane Oxygenation. Semin Thromb Hemost. 2018;44:20-9. doi:10.1055/s-00371606179

24. Sy E, Sklar MC, Lequier L, Fan E, Kanji HD. Anticoagulation practices and the prevalence of major bleeding, thromboembolic events, and mortality in venoarterial extracorporeal membrane 
oxygenation: A systematic review and meta-analysis. J Crit Care. 2017;39:87-96. doi:10.1016/j.jcrc.2017.02.014

25. Murphy DA, Hockings LE, Andrews RK, Aubron C, Gardiner EE, Pellegrino VA, et al. Extracorporeal membrane oxygenation-hemostatic complications. Transfus Med Rev. 2015;29:90-101. doi:10.1016/j.tmrv.2014.12.001

26. Anselmi A, Guinet P, Ruggieri VG, Aymami M, Lelong B, Granry S, et al. Safety of recombinant factor VIla in patients under extracorporeal membrane oxygenation. Eur $\mathrm{J}$ Cardio-thoracic Surg. 2016;49:78-84. doi:10.1093/ejcts/ezv140

27. Kerbaul F, Gariboldi V, Giorgi R, Mekkaoui C, Guieu R, Fesler P, et al. Effects of levosimendan on acute pulmonary embolism-induced right ventricular failure. Crit Care Med. 2007;35:1948-54. doi:10.1097/01.CCM.0000275266.33910.8D

28. Hu Y, Li Q, Wei BG, Zhang X Sen, Torsha TT, Xiao J, et al. Blood loss of total knee arthroplasty in osteoarthritis: An analysis of influential factors. J Orthop Surg Res. 2018;13:325. doi:10.1186/s13018-018-1038-0

29. Fiumara K, Kucher N, Fanikos J, Goldhaber SZ. Predictors of major hemorrhage following fibrinolysis for acute pulmonary embolism. Am J Cardiol. 2006;97:127-9. doi:10.1016/j.amjcard.2005.07.117

30. Kuo WT, Banerjee A, Kim PS, De Marco FJ, Levy JR, Facchini FR, et al. Pulmonary embolism response to fragmentation, embolectomy, and catheter thrombolysis (PERFECT): Initial results from a prospective multicenter registry. Chest. 2015;148:667-73. doi:10.1378/chest.15-0119

31. Tapson VF, Sterling K, Jones N, Elder M, Tripathy U, Brower J, et al. A Randomized Trial of the Optimum Duration of Acoustic Pulse Thrombolysis Procedure in Acute Intermediate-Risk Pulmonary Embolism: The OPTALYSE PE Trial. JACC Cardiovasc Interv. 2018;11:1401-10. doi:10.1016/j.jcin.2018.04.008

32. Piazza G, Hohlfelder B, Jaff MR, Ouriel K, Engelhardt TC, Sterling KM, et al. A Prospective, Single-Arm, Multicenter Trial of Ultrasound-Facilitated, Catheter-Directed, Low-Dose Fibrinolysis for Acute Massive and Submassive Pulmonary Embolism: The SEATTLE II Study. JACC Cardiovasc Interv. 2015;8:1382-92. doi:10.1016/j.jcin.2015.04.020

33. Kucher N, Boekstegers P, Müller OJ, Kupatt C, Beyer-Westendorf J, Heitzer T, et al. Randomized, controlled trial of ultrasound-assisted catheter-directed thrombolysis for acute intermediate-risk pulmonary embolism. Circulation. 2014;129:479-86. doi:10.1161/CIRCULATIONAHA.113.005544

34. Owens CA. Ultrasound-enhanced thrombolysis: EKOS endo wave infusion catheter system. Semin Intervent Radiol. 2008;25:37-41. doi:10.1055/s-2008-1052304

35. Kaymaz C, Akbal OY, Tanboga IH, Hakgor A, Yilmaz F, Ozturk S, et al. Ultrasound-Assisted CatheterDirected Thrombolysis in High-Risk and Intermediate-High-Risk Pulmonary Embolism: A MetaAnalysis. Curr Vasc Pharmacol. 2017;16:179-89. doi:10.2174/1570161115666170404122535

36. Ruohoniemi DM, Sista AK, Doany CF, Heerdt PM. Perioperative pulmonary thromboembolism: Current concepts and treatment options. Curr Opin Anaesthesiol. 2018;31:75-82.

doi:10.1097/AC0.0000000000000550

Page $12 / 16$ 
37. Yamada K, Tsuji H, Kimura S, Kato H, Yano S, Ukimura N, et al. Effects of argatroban and heparin on thrombus formation and tissue plasminogen activator-induced thrombolysis in a microvascular thrombosis model. Thromb Res. 2003;109:55-64. doi:10.1016/S0049-3848(03)00105-1

38. Bethea BT, Elliot JW, Richardson JB, Ahmed MI. Treatment of pulmonary embolism with argatroban and ultrasound-assisted catheter-directed thrombolysis with alteplase in a patient with heparininduced thrombocytopenia. Am J Heal Pharm. 2017;74:1153-7. doi:10.2146/ajhp160368

\section{Figures}

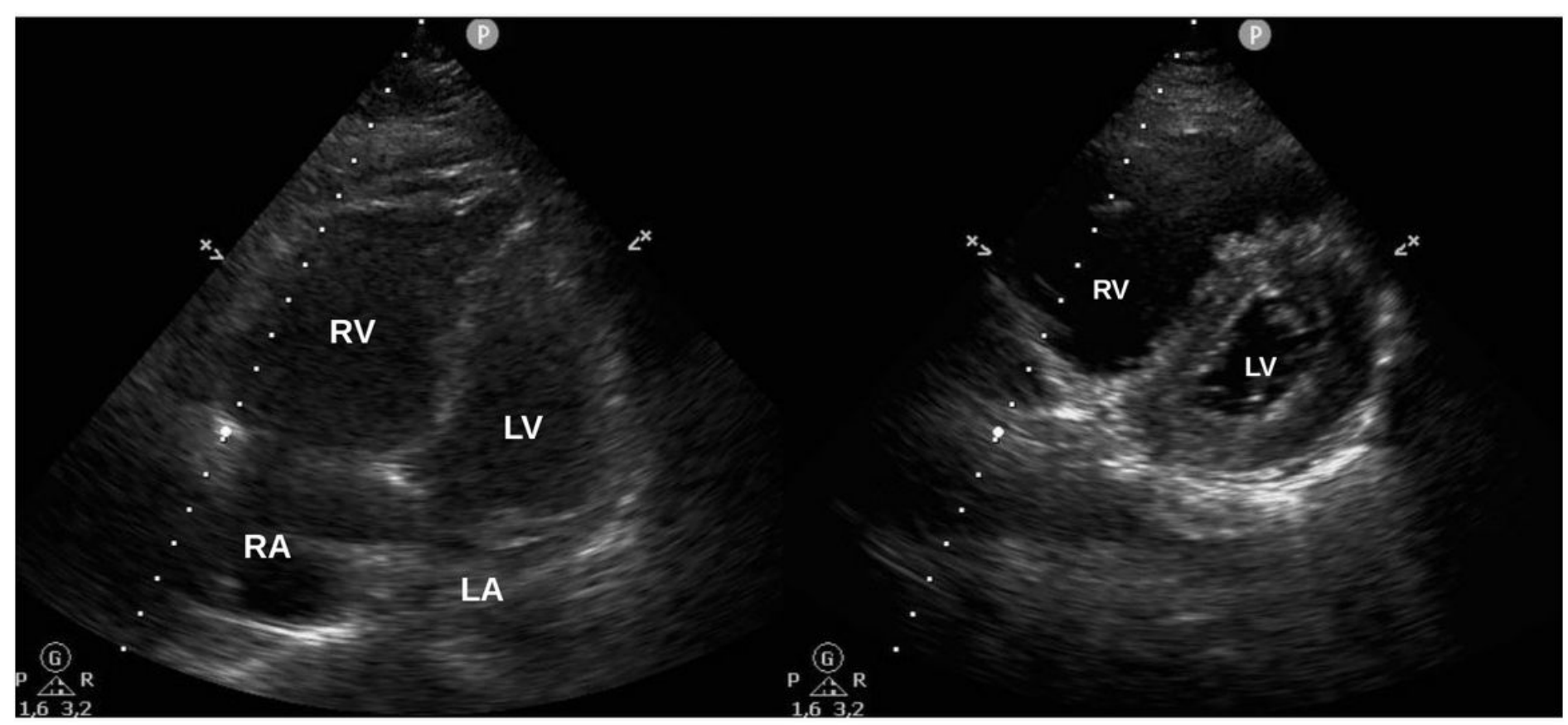

\section{Figure 1}

(a) Apical four chamber view showed severe RV dilatation and (b) parasternal short axis view revealed flattened interventricular septum ( $L A=$ left atrium, $R A=$ right atrium, $L V=$ left ventricle, $R V=$ right ventricle) 


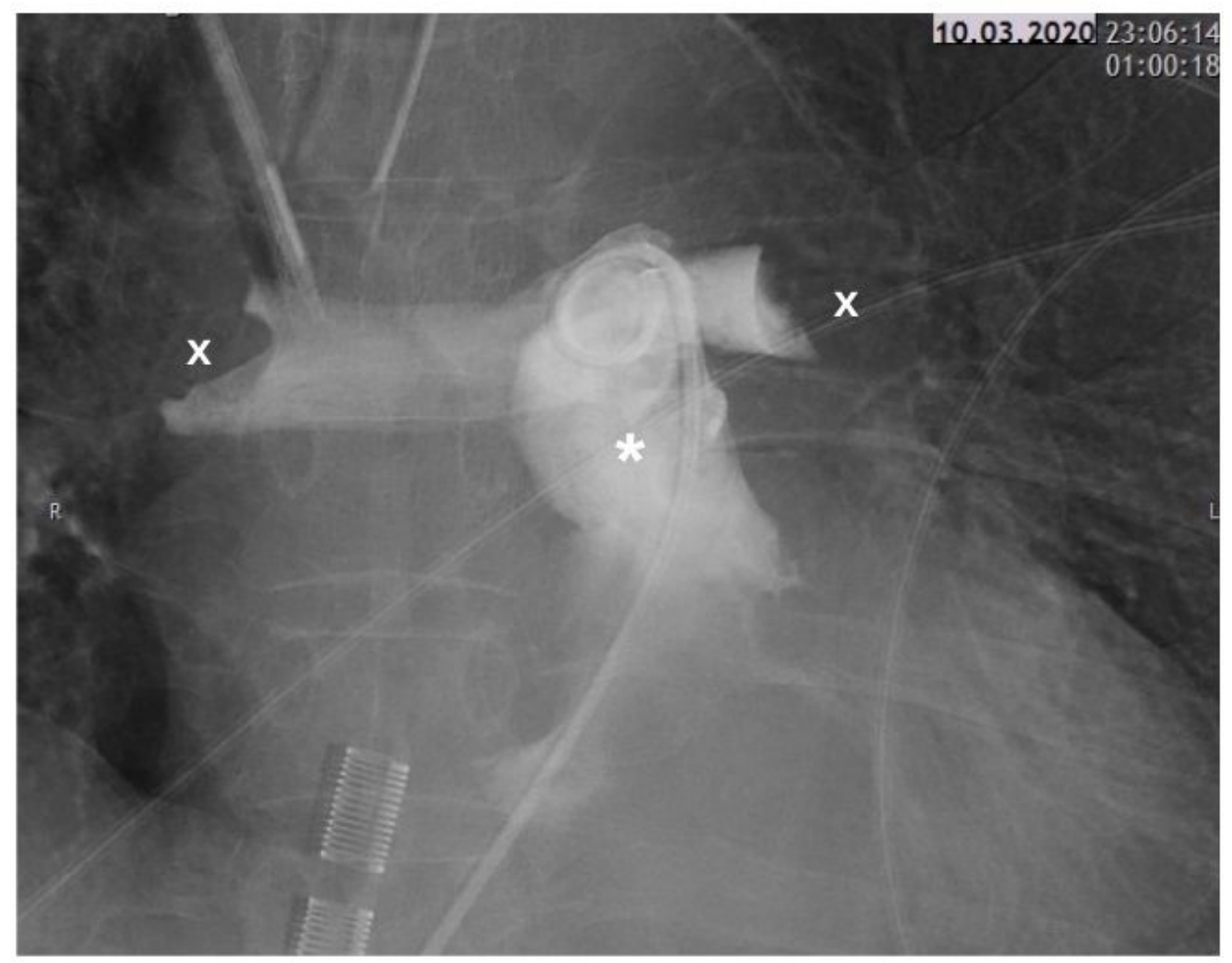

Figure 2

Angiography showed bilateral occlusion of both main pulmonary arteries $(*=$ pulmonary trunk, $x=$ thrombotic burden) 


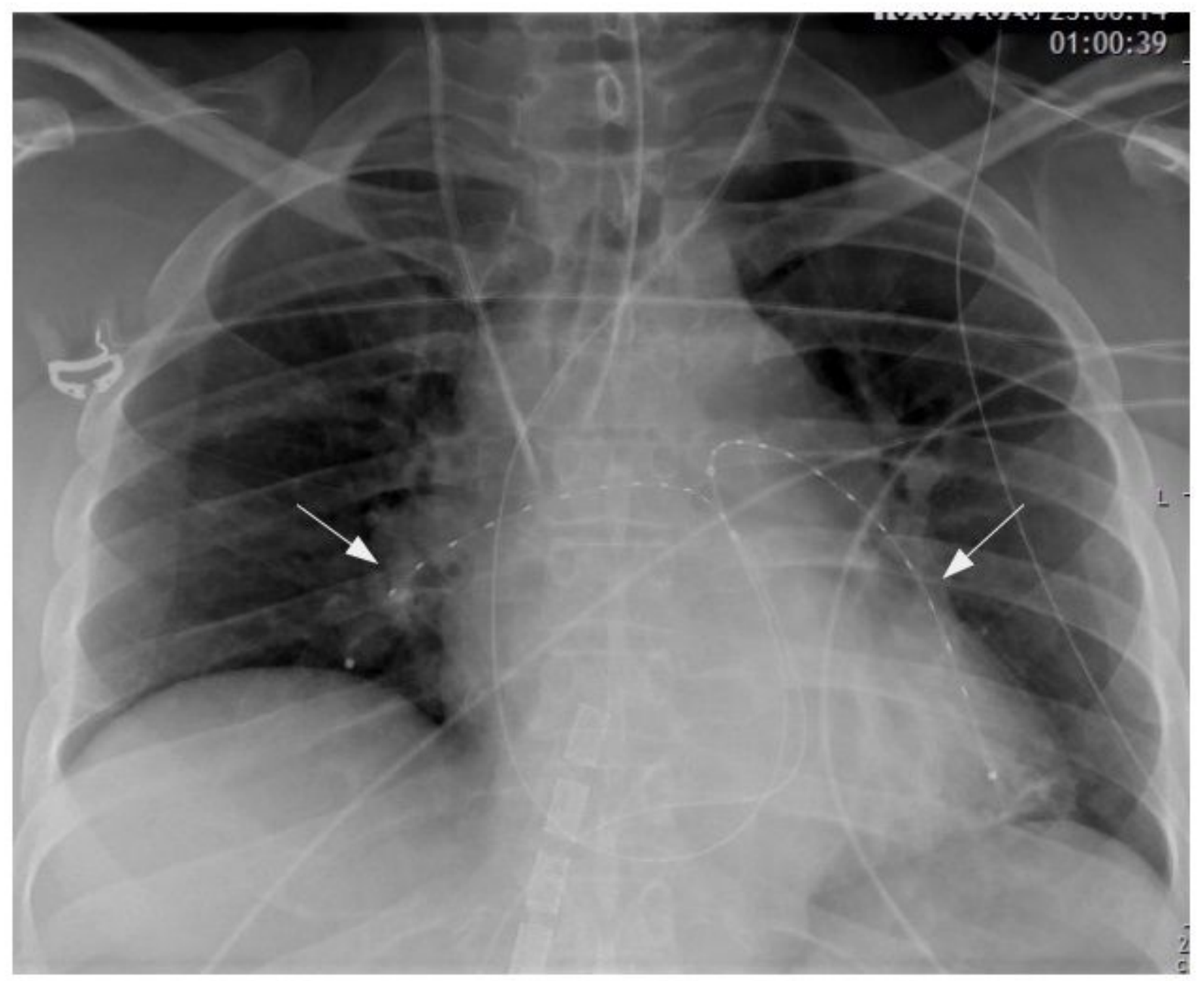

Figure 3

Chest X-ray shows both catheters in situ (arrows) 


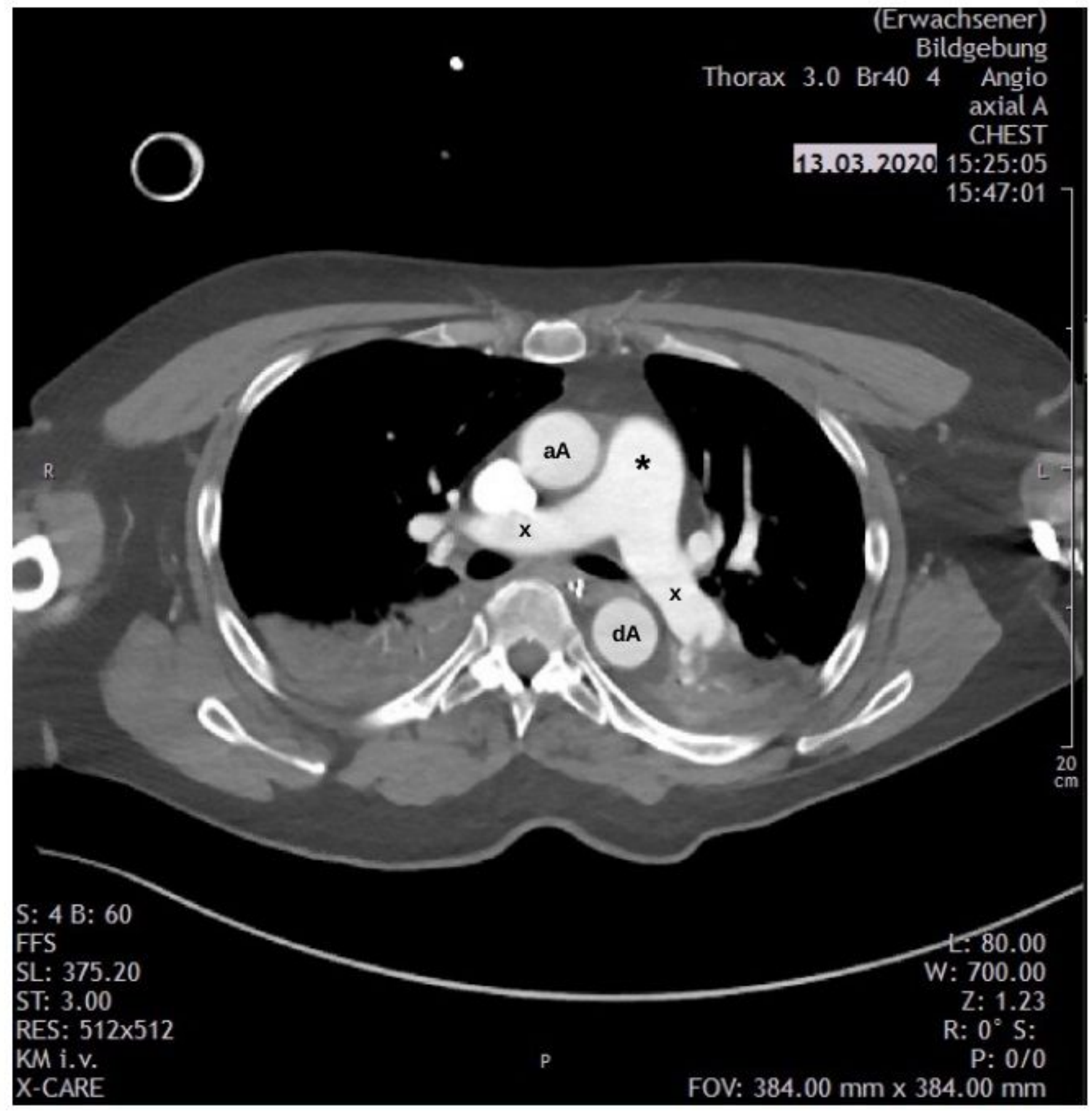

\section{Figure 4}

CT scan revealed regredient embolic burden $(\mathrm{x})$ and improved recanalization after ECMO explantation ${ }^{*}=$ pulmonary trunk, aA = ascending aorta, $\mathrm{dA}=$ descending aorta) 